\title{
Carbon Steel Versus Stainless Steel in the Design of Electrode Arms for UHP Electric Arc Furnaces
}

\author{
Peter F. Ryff
}

Ryerson University

\section{Vladimir Bulat}

EMPCO (Canada) Ltd

digital.library.ryerson.ca/object/64

Please Cite:

Ryff, P. F., \& Bulat, V. (1981). Carbon steel versus stainless steel in the design of electrode arms for UHP electric arc furnaces. IEEE Transactions on Industry Applications, IA-17(2), 222-230.

doi:10.1109/TIA.1981.4503929

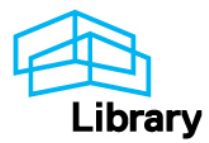




\title{
Steel Versus Stainless Steel in the Design of Electrode Arms for UHP Electric Arc Furnaces
}

\author{
PETER F. RYFF, SENIOR MEMBER, IEEE, AND VLADIMIR BULAT
}

\begin{abstract}
The operating temperatures due to eddy current induced heating effects in water-cooled electrode arms of three-phase ultrahigh powered (UHP) electric arc furnaces during operation are calculated. The selection of commonly used materials (carbon steel SAE 1010 and stainless steel - 304) is examined with regard to optimum cooling water flow and safe allowable temperature increases. Calculated results are substantiated by temperature measurements conducted on the center-phase electrode arm and its water cooling system of a recently installed furnace in the field.
\end{abstract}

\section{INTRODUCTION}

D URING the last few years many arc furnaces have been upgraded for much higher production rates by replacing the furnace transformers, using larger diameter electrodes and modifying the secondary circuits from transformer to electrode clamps. Although significant benefits were derived from this, it also has created many problems concerning magnetically induced power losses due to eddy currents, causing overheating. With increases in electrode currents of 100 percent or more in the case of upgrading, as well as in the design of new ultrahigh powered (UHP) electric arc furnaces, significantly higher power input levels have been reached. This meant that extra precautions had to be taken, since the conventional furnaces were built for relatively small currents compared with UHP operations. Therefore, the electrode arms are exposed to much stronger magnetic fields than before and serious heating problems could result. In addition, restrictions on geometrical arrangements are imposed by the mel-

Paper IUSD 80-15, approved by the Electric Process Heating Committee of the IEEE Industry Applications Society for publication in this TRANSACTIONS. Manuscript released for publication July 1, 1980

P. F. Ryff is with the Ryerson Poly technical Institute, 50 Gould St., Toronto, ON, Canada M5B $1 \mathrm{E} 8$.

V. Bulat is with EMPCO (Canada) Ltd., Oshawa, ON, Canada. shop in their request for available height and space around a furnace, thereby limiting the spacing of conductors away from steel components.

Often the problem was solved by using stainless steel instead of carbon steel electrode arms. Its negligible mag. netic permeability and lower electrical conductivity inherently limits the eddy currents, hence the resulting heating effects. However, the penetration of the magnetic field at the prevailing frequency $(60 \mathrm{~Hz})$ is more complete in stainless steel. This means that although the heat generation is lower, it is fairly constant throughout the material. With carbon steel it is concentrated mainly in a thin layer, but it has a better thermal conductivity, which implies that any induced heat is extracted more readily by the coolant.

Considering the electrical and thermal properties of the materials involved, it was not apparent that the use of stainless steel necessarily meant a satisfactory electrode arm operating temperature for a particular furnace design. In addition, due to the dramatic price increases of stainless steel as compared to carbon steel, the electrode arm production costs are considerably higher, thereby influencing furnace economy.

To answer the basic question of material selection for a specific design and to isolate critical design parameters, as well as to aid in future furnace designs, this investigation was carried out. The electrode arm operating temperatures, during and under the most severe furnace conditions, were determined for a three-phase electric arc furnace design using these materials in the design of the electrode arms. Factors such as coolant flow and thickness of material were also considered.

The particular furnace studied was recently upgraded to produce 450000 tons of steel per year. Originally built with a 19-ft 0-in inside diameter (ID) shell having a standard refractory, 20-in (diameter) electrodes, and powered with a three- 
phase 53 MVA furnace transformer was modified to a $20-\mathrm{ft}$ 0 -in ID shell with a water-cooled shell and roof, 24-in electrodes and supplied by a 93 MVA transformer. To accept the 24-in diameter electrodes and significantly larger electrical and mechanical loading, all furnace components (except rockers, furnace bottom, and platform) were redesigned and modified to meet the new design criteria. New electrode arms, bus bar designs, and the selection of electrode arm material was a prime consideration in view of the above mentioned concerns.

The result of this study was a deciding factor in the material selection of the electrode arms and is subsequently presented. After presenting the theoretical results of this study, temperature measurements on the actual furnace center electrode arm and its water cooling system are included, verifying the theoretically predicted values on this UHP furnace, operating satisfactorily since midsummer 1979.

\section{FORMULATION OF THE PROBLEM}

The general assembly of the furnace investigated is illustrated in Fig. 1, showing the center electrode arm with the bus tubes and its support structure. There are three such electrode arms, one per phase, each in turn supported by an electrode mast (column) which is hydraulically operated to raise and lower the entire electrode arm assembly. The electrode arms support the current carrying water-cooled bus tubes in such a way that the center bus tube is raised to provide triangulation with those of the two longer outside ones. The electrodes protrude through the furnace roof onto the charging material and are arranged such that they form an equilateral triangle on the electrode pitch circle as illustrated in Fig. 2. It shows a top view of the furnace with extraneous assemblies, such as the roof swing support system (gantry arms) deleted for clarity. To aid in the analysis and because of the complex geometrical configuration of the electrode arms and auxiliary components, making an exact analysis impossible, an as simple and fundamental a geometry as possible is shown in Fig. 3, while still retaining the configuration of a particular design. It represents a cross section of the electrode arms indicating their construction and at the same time illustrates the triangulation of the bus tube system. There are two current buses per phase, each carrying half the electrode current, the center arm being the center phase of the supply.

The triangulation throughout of the current supply system from the transformer delta closure to the electrodes is desired from an electrical point of view. It maintains the secondary impedance balanced as much as possible on a per phase basis by equalizing induced voltages. While this position is desirable from a power input and phase balance standpoint, it must be recognized that especially during furnace startup and the initial melt down stages this condition is seldom met in practice. This is because of different electrode lengths and uneven charging material levels under the electrodes.

Fig. 4 and 5 schematically show possible relative elevations of the electrode arms with respect to each other from the desired position with regard to equilateral triangulation. The raised position of the center electrode arm, as indicated in Fig. 5, represents the worst possible condition from the

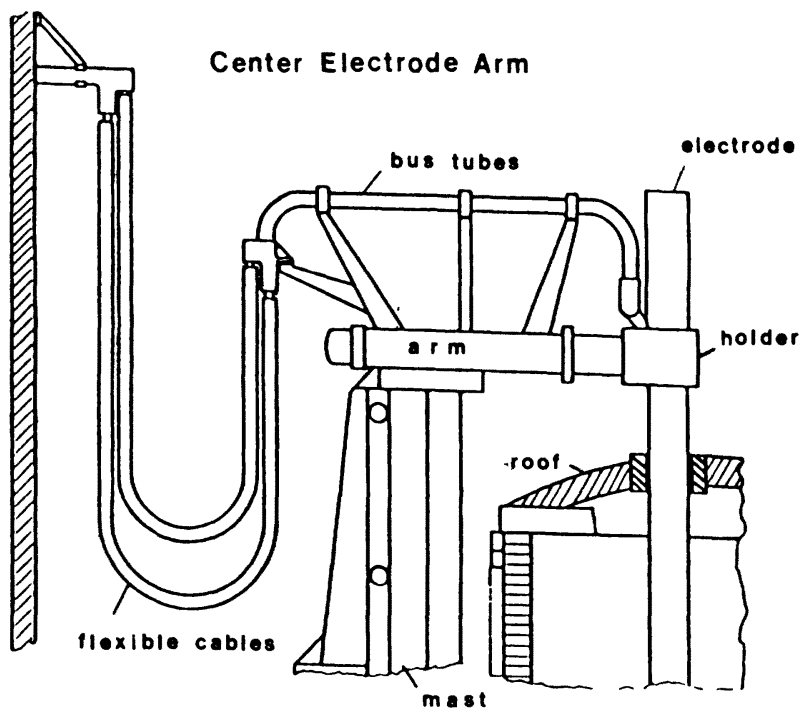

Fig. 1. Illustration of center electrode arm assembly in electric arc furnace.

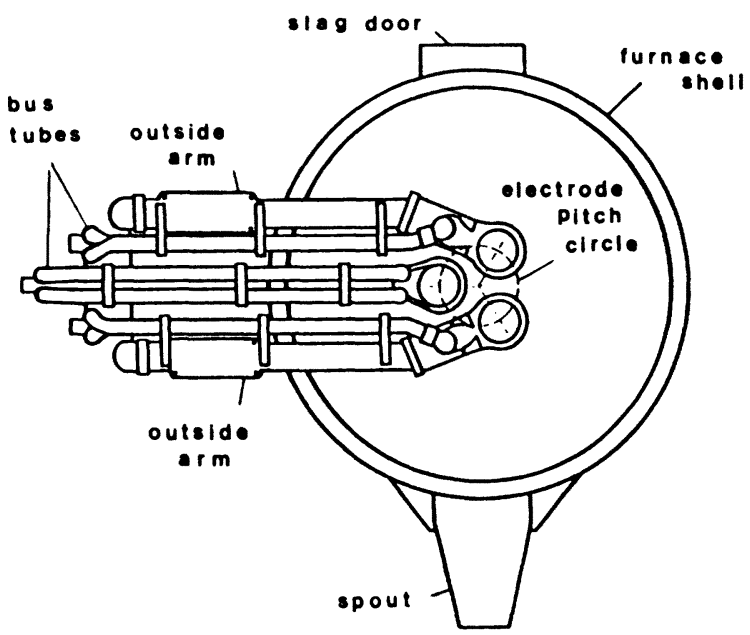

Fig. 2. Top view of electric arc furnace.

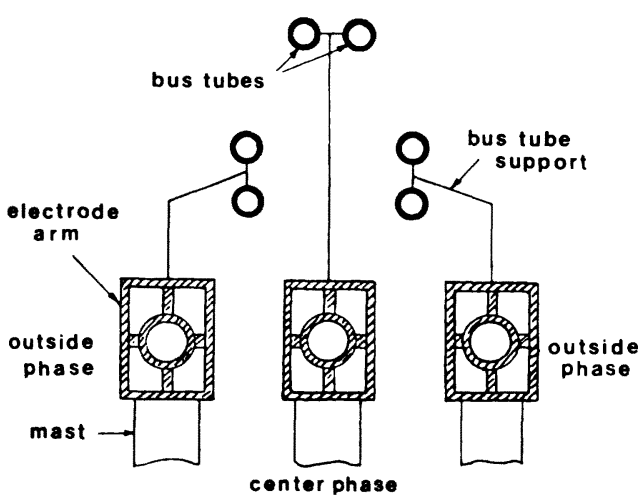

Fig. 3. End elevation bus tubes triangulated.

point of view of induced heating. It is also apparent that in this position the presence of the outside arms is of negligible influence as far as the effect on the induced losses in the center is concerned [1]. Hence their effect on the center arm induced eddy current losses is assumed negligible in the calculations. This assumption is further substantiated from 


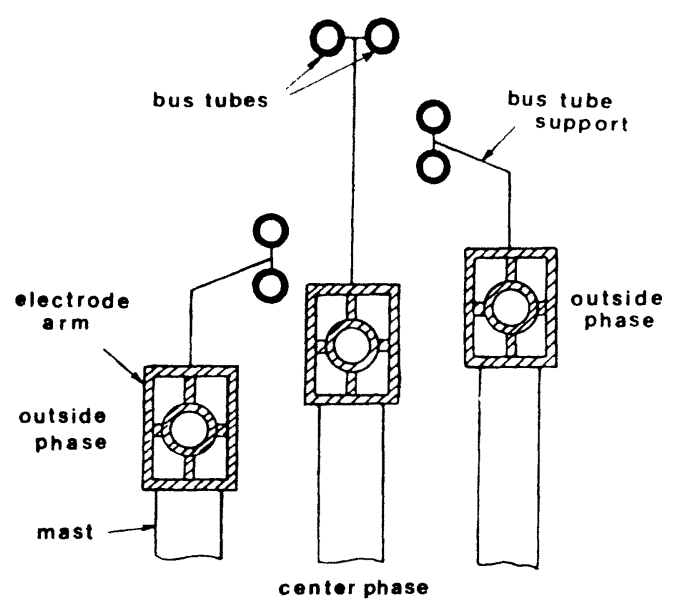

Fig. 4. End elevation bus tubes center and right outside phase raised.

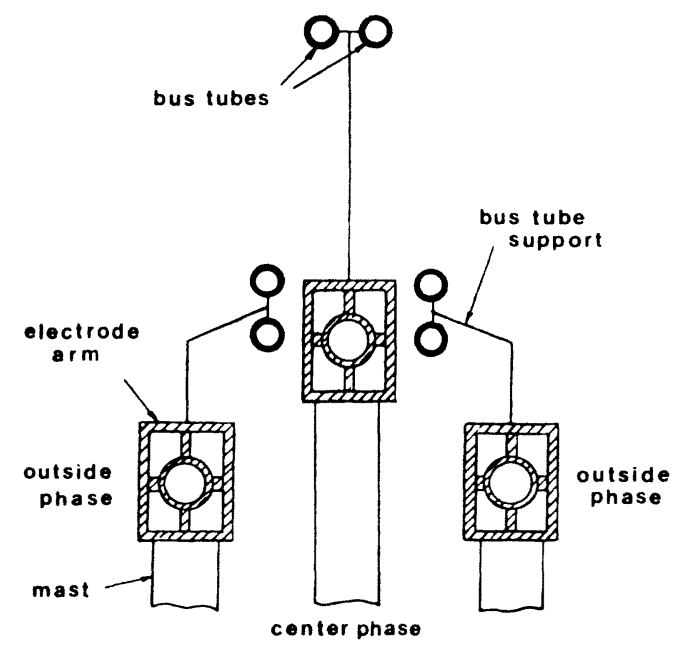

Fig. 5. End elevation bus tubes center phase raised.

practical observations and measurements, which indicate that the outside electrode arms operate at temperatures considerably lower than those of the center arm in all circumstances.

\section{POWER LOSS CALCULATION}

Referring to Fig. 3, the basic configuration consists of three pairs of bus tubes initially in the geometric arrangement indicated. During furnace operation, in the event where the center arm is raised from the desired position in relation to the outside arms, the induced eddy currents and subsequently the power losses giving rise to heating effects in the center arm become progressively worse. This can readily be seen in Fig. 5, where the center arm is adjacent to the outside bus tubes.

Since the superposition theorem can be applied to the magnetic fields, the induced losses due to a single conductor is examined first. The resulting expression is then extended to account for all six conductors, properly accounting for their relative position in space around the center arm and their phase sequence of the supply frequency.

Referring to Fig. 6, a single conductor is shown adjacent to the electrode arm, at first assumed to be of circular geo-

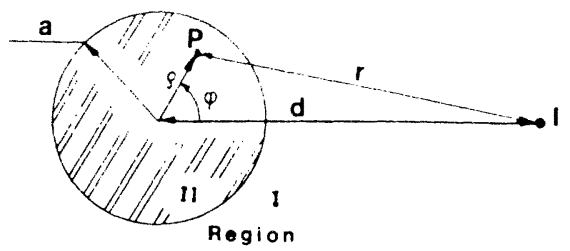

Fig. 6. Single bus tube adjacent to circular electrode arm body.

metry. The conductor carries an ac current $I$, placed at the distance $d$ indicated. The arm has a radius $a$ and its material permeability $\mu=\mu_{0} \mu_{r}$, where $\mu_{0}=4 \pi \times 10^{-7} \mathrm{H} / \mathrm{m}$ and $\mu_{r}$ the relative permeability. The material electrical conductivity is taken as $\sigma$. For the purpose of the analysis, end-effects are neglected; this implies that the electric and magnetic fields are independent of the cylindrical coordinate $(\xi)$ perpendicular to the plane of the paper, reducing the problem to a twodimensional one. In addition, hysteresis and saturation phenomena are not included. The power loss density $P_{v}$ at any point inside the material depends on the magnitude of the magnetic vector potential $\bar{A}^{2}$, namely

$$
P_{v}=\sigma \bar{E}^{2}=\sigma|-i \omega \bar{A}|^{2}=\sigma \omega^{2}|\bar{A}|^{2}
$$

where $\omega$ is the radial frequency of the supply current. The potential $\bar{A}$ is obtained by solving the equation

$$
\nabla^{2} A=0
$$

outside the material (region I) and

$$
\nabla^{2} A=i k^{2} A
$$

inside (region II), see Fig. 6. It can be shown that the solution of these equations using cylindrical coordinates is [3], in region $\mathrm{I}$,

$$
\bar{A}_{1}=\frac{\mu_{0} I}{2 \pi} \ln r+\mu \sum_{n=1}^{\infty} C_{n} \rho^{-n} \cos n \varphi
$$

and in region II

$$
\bar{A}_{2}=\sum_{n=1}^{\infty} \mu D_{n} J_{n}(k \rho) \cos n \varphi
$$

where $k^{2}=\omega \mu \sigma, J_{n}$ is the Bessel function of order $n$ and

$$
r=\left(d^{2}+\rho^{2}-2 d \rho \cos \varphi\right)^{1 / 2}
$$

being the radial distance to the point where the potential is calculated which makes an angle $\varphi$ with the reference axis. The unknown integration constants $C_{n}$ and $D_{n}$ can be determined by solving the boundary problem at $\rho=a$, which involves equating the tangential and normal components of the magnetic field, namely [4]

$$
-\frac{1}{\mu_{1}} \frac{\partial A_{1}}{\partial \rho}=-\frac{1}{\mu_{2}} \frac{\partial A_{2}}{\partial \rho} \text { (tangential) }
$$


and

$$
\frac{\partial A_{1}}{\partial \varphi}=\frac{\partial A_{2}}{\partial \varphi} \text { (normal) }
$$

Carrying out the indicated derivations, equating components of each value of $n$ and making some simplifying substitutions, the constants are determined. If the so obtained value of $D_{n}$ is substituted into equation (5), the following expression results [5] in

$$
A_{2}=-\frac{\mu I}{\pi} \sum_{n=1}^{\infty}\left(\frac{a}{d}\right)^{n} \frac{J_{n}(k \rho)}{n\left(\mu_{r}+1\right) J_{n}-k a J_{n+1}} \cos n \varphi
$$

where the Bessel functions have the argument $k a$ if not explicitly specified. Consider now the complete arrangement as shown in Fig. 7. Each bus tube carries half of the corresponding phase current. Taking into account their space location and electrical phase sequence, the total expression for the vector potential inside the material (by extending the previous analysis and using the superposition principle) becomes

$$
\begin{aligned}
A= & -\frac{\mu I}{2 \pi} \sum^{\infty} \frac{J_{n}(k \rho)}{n\left(\mu_{r}+1\right) J_{n}-k a J_{n+1}} \\
& \cdot\left\{\left(\frac{a}{d_{2}}\right)^{n} \cos n\left(\varphi-\theta_{2}\right)+\left(\frac{a}{d_{3}}\right)^{n} \cos n\left(\varphi-\theta_{3}\right)\right. \\
& +\left(\frac{a}{d_{1}}\right)^{n}\left(-\frac{1}{2}-i \frac{\sqrt{3}}{2}\right) \\
& \cdot\left[\cos n\left(\varphi-\theta_{1}\right)+\cos n\left(\varphi+\theta_{1}\right)\right] \\
& +\left(-\frac{1}{2}+i \frac{\sqrt{3}}{2}\right)\left[\left(\frac{a}{d_{2}}\right)^{n} \cos n\left(\varphi+\theta_{2}\right)\right. \\
& \left.\left.+\left(\frac{a}{d_{3}}\right)^{n} \cos n\left(\varphi+\theta_{3}\right)\right]\right\}
\end{aligned}
$$

where the angle $\theta$ refers to the angular displacement of the bus tubes with respect to the reference axis and $\varphi$ the angle at which the vector potential is calculated in the material at the radial distance $\rho$ from the center. All other symbols used are as explained or using the notation in Fig. 7.

The transition from the circular electrode arm in Fig. 6 to the rectangular one in Fig. 7 should be noted. It is assumed that the rectangular geometry of the arm does not influence the basic expression (6) for the vector potential, which strictly speaking is correct only for the circular geometry. However, this assumption seemed realistic in view of all other necessary practical simplifications made and proved to be justified in the subsequent work carried out. When calculating the vector potential at any point $P$ in the material of the rectangular geometry, its "effective" circular radius $a$ associated with that point must then be determined. It is represented by the distance $S C$ in Fig. 8 (for an arbitrary field point $P$ ), measured

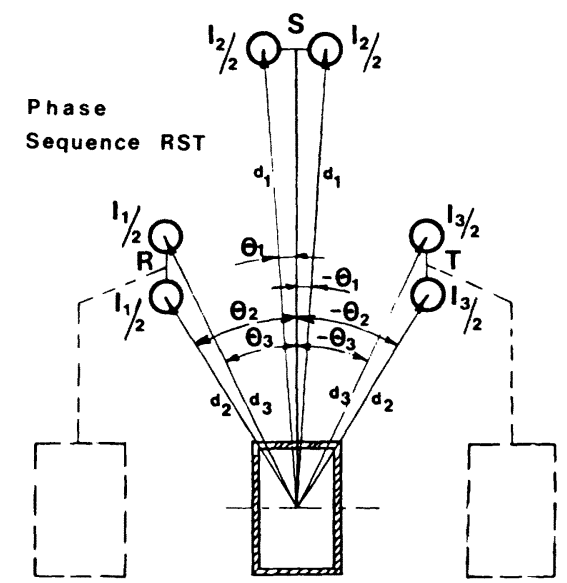

Fig. 7. Center electrode arm and bus bar arrangement in triangulated position.

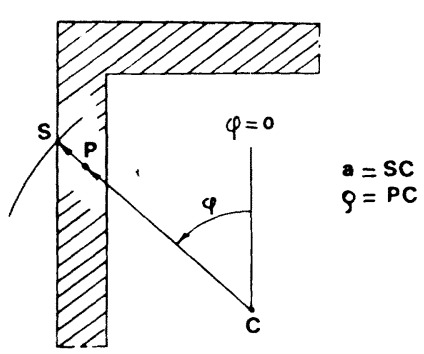

Fig. 8. Determination of effective radius $a$, associated with a field point $P(\rho, \varphi)$.

along the radial distance vector $\rho=P C$. When the point is located on the outside surface, $a=\rho=S C$. The angle $\varphi$ being the angular coordinate of the field point is readily obtained in each instance. Knowing the vector potential $\bar{A}$ at the point enables the calculation of the power loss density at that location by the use of (1).

\section{TEMPERATURE DISTRIBUTION}

Having determined the induced losses throughout the electrode arm material in the above manner, the task remains to relate this into expected temperature increases. In Fig. 9, the electrode arm construction is illustrated showing the water cooling paths. Because of the relatively thin wall thickness of the electrode arm body and good thermal conductivity of the material, the heat flow is taken to be one-dimensional across the plate material. This is an accurate assessment since the temperature difference between outside (furnace atmosphere) and inside (coolant) boundaries is relatively large. Also, the induced losses vary greatly in this direction since the field decays rapidly inside the material. Therefore, the temperature calculation reduces to that of a plate with forced water cooling on one side and convective and conductive air cooling on the other. The induced power loss inside the material is represented by the internal heat generation term $q^{\prime \prime \prime}$, which is nonlinear. Finally, an external heat flux due to radiation from the hot furnace roof is considered. This is done by calculating the radiant energy transfer between the surfaces in question and comparing this to the convective and conductive heat transfer across the boundary. Knowing these fluxes, the 


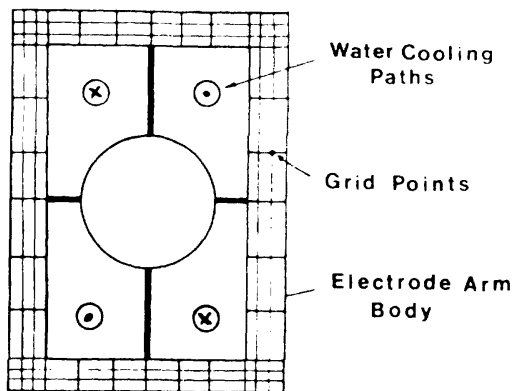

Fig. 9. Electrode arm cross section showing mesh points used in calculating power losses and temperatures.

surface heat transfer coefficient $h$ is adjusted to incorporate this effect.

Equating the heat flow in the fluids to that of the solid, the appropriate boundary conditions are obtained. In general this leads to

$$
\pm k\left(\frac{\partial T}{\partial n}\right)=h\left(T-T_{f}\right)
$$

where $k$ is the thermal conductivity of the material and $T_{f}$ is the average fluid bulk temperature. If the outward surface normal is in the negative $x$ direction, see Fig. 10, then

$$
\frac{\partial T}{\partial n}=-\frac{\partial T}{\partial n}
$$

The heat equation to be solved, considering the internal heat generation in the steady-state, becomes

$$
\frac{d^{2} T}{d x^{2}}+\frac{q^{\prime \prime \prime}}{k}=0 .
$$

The following boundary conditions prevail [6]: outside, $x=0$,

$$
k \frac{d T(0)}{d x}=h_{o}\left[T(0)-T_{o}\right],
$$

inside,

$$
x=1, \quad-k \frac{d T(1)}{d x}=h_{i}\left[T(1)-T_{i}\right] .
$$

The subscripts $o$ and $i$ refer to the outside and inside boundaries, respectively; $q^{\prime \prime \prime}=q_{0}{ }^{\prime \prime \prime} e^{-x / \delta}$, where $q_{0}{ }^{\prime \prime \prime}$ is the induced power loss density at the surface and $\delta=\left(2 / \mu_{0} \omega \sigma\right)^{1 / 2}$ is the penetration depth of the magnetic field at the working frequency.

Solving the differential equation and applying the boundary conditions yields the solution of the temperature $T$ throughout the material having a thickness $l$. The solution after a somewhat lengthy but straightforward derivation is

$$
T=-\delta \phi e^{-x / \delta}+C_{1} x+C_{2}
$$

where

$$
C_{1}=\frac{-\phi \alpha-h_{o} h_{i} / k\left(T_{o}-T_{i}\right)}{h_{i}+h_{o}\left(1+h_{i} l / k\right)}
$$

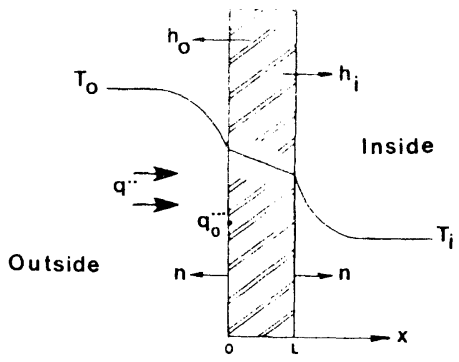

Fig. 10. Heat conduction in plane wall with internal heat generation and forced convection inside.

$$
C_{2}=\frac{\phi \beta+h_{o}\left(1+h_{i} l / k\right) T_{o}+h_{i} T_{i}}{h_{i}+h_{o}\left(1+h_{i} l / k\right)}
$$

and

$$
\begin{aligned}
& \alpha=h_{i}\left(1+h_{o} \delta / k\right)+h_{o}\left(1-h_{i} \delta / k\right) e^{-l / \delta} \\
& \beta=\left(k+h_{o} \delta\right)\left(1+h_{i} l / k\right)-\left(k-h_{i} \delta\right) e^{-l / \delta} \\
& \phi=\frac{\delta q_{0}{ }^{\prime \prime \prime}}{k} .
\end{aligned}
$$

\section{Surface Heat Transfer Coefficients}

To determine the temperature distribution in the watercooled electrode arm, it is necessary to determine the surface heat transfer coefficients $h_{i}$ and $h_{o}$ for the solution of (12). For the flow inside the duct, see Fig. 9, knowing the given geometry and flow condition, the following emperical equation appropriate for the configuration was selected in terms of a Nusselt number, namely [7]

$$
\mathrm{Nu}_{D_{H}}=0.023 /\left(\operatorname{Re}_{D_{H}}{ }^{0.2} \operatorname{Pr}^{0.67}\right)
$$

where the symbols Re and Pr denote the Reynolds and Prandtl number, respectively. The significant length in the Nusselt number, as in the Reynold number, is the hydraulic diameter $D_{H}$ defined as

$$
D_{H}=4 \frac{\text { flow cross sectional area }}{\text { wetted perimeter }}
$$

The Reynolds number, characterizing the flow condition, is obtained from

$$
\operatorname{Re}_{D_{H}}=\frac{\rho D_{H} V}{u}
$$

in which $\rho$ is the density, $V$ is the velocity and the ratio $u / \rho$ is the kinematic viscosity of the fluid. The Prandtl number is a function of the fluid property alone, relating the temperature to the velocity distribution and is defined at the bulk temperature as

$$
\operatorname{Pr}_{b}=\frac{c u}{k}
$$

where $c$ is the specific heat and $u$ is the absolute viscosity of the fluid, in this instance, water. Having obtained the characteristic numbers describing the flow conditions, the heat transfer coefficient $h_{i}$ can be calculated from the relation

$$
\mathrm{Nu}_{D_{H}}=\frac{h_{i}}{c \rho V}
$$


relating the Nusselt number to the heat transfer for the fluid in the conduit. The numerical value of $h_{i}$ depends on the choice of reference temperature in the fluid; however, it is common practice as one which is followed here to use the average bulk temperature of the fluid as a reference.

The remaining quantity to be obtained is the heat transfer coefficient $h_{o}$ at the outside boundary. At this boundary there is a combined effect of the heat flow due to the hot ambient temperature $T_{a}$ as well as receiving a net radiant heat flux $q^{\prime \prime}$ due to radiation from the furnace roof. These combined effects can be determined by

$$
\pm k \frac{\partial T}{\partial n}+q^{\prime \prime}=h\left(T_{s}-T_{a}\right)
$$

or

$$
\pm k\left(\frac{\partial T}{\partial n}\right)=h_{o}\left(T_{s}-T_{a}\right)
$$

where $h_{o}=h_{c}+h_{r}$ is the combined heat transfer coefficient due to convection and radiation and $T_{S}$ is the surface temperature.

Equations (18) and (19) are independent of the actual temperature distribution. Initially $h_{c}$ can be estimated with the help of published data and corrected afterwards, if necessary, to suit the prevailing conditions at the boundary once the surface temperature is known. The value of $h_{r}$ can be estimated using techniques which have evolved for the heat transfer rates resulting from thermal radiation between surfaces [8]. Factors such as the radiation characteristic, geometries, and orientation of the surfaces with respect to one another are considered.

\section{NUMERICAL RESULTS}

\section{Power Loss Density}

The method developed was applied to the particular furnace design proposed having a maximum operating current of $80000 \mathrm{~A}$. To obtain the power loss density distribution throughout the material, the arm cross section was divided by a mesh as schematically indicated in Fig. 9. A computer program was developed to obtain the power loss density at the nodal points throughout the cross section. The center arm was raised from the initially triangulated position and the losses calculated for each incremental step in center electrode arm elevation to the expected worst condition. Some computer calculated results for the center electrode arm using carbon steel (SAE 1010) material are shown in Fig. 11 and 12 for the selected center arm elevation $h$ indicated. The power loss density ranges represented by the numbers are given in Table I. Recalculating the losses when using stainless steel (304) material gives the results shown in Fig. 13 and 14 for identical center arm elevations. The influence of the materials used is apparent when comparing the results. Also, the effect on the loss distribution is clearly illustrated when the center arm is raised. Resulting temperatures calculated are presented next.

\section{Temperature Calculations}

When the center electrode arm is raised in the power loss calculations, the corresponding temperature distribution at

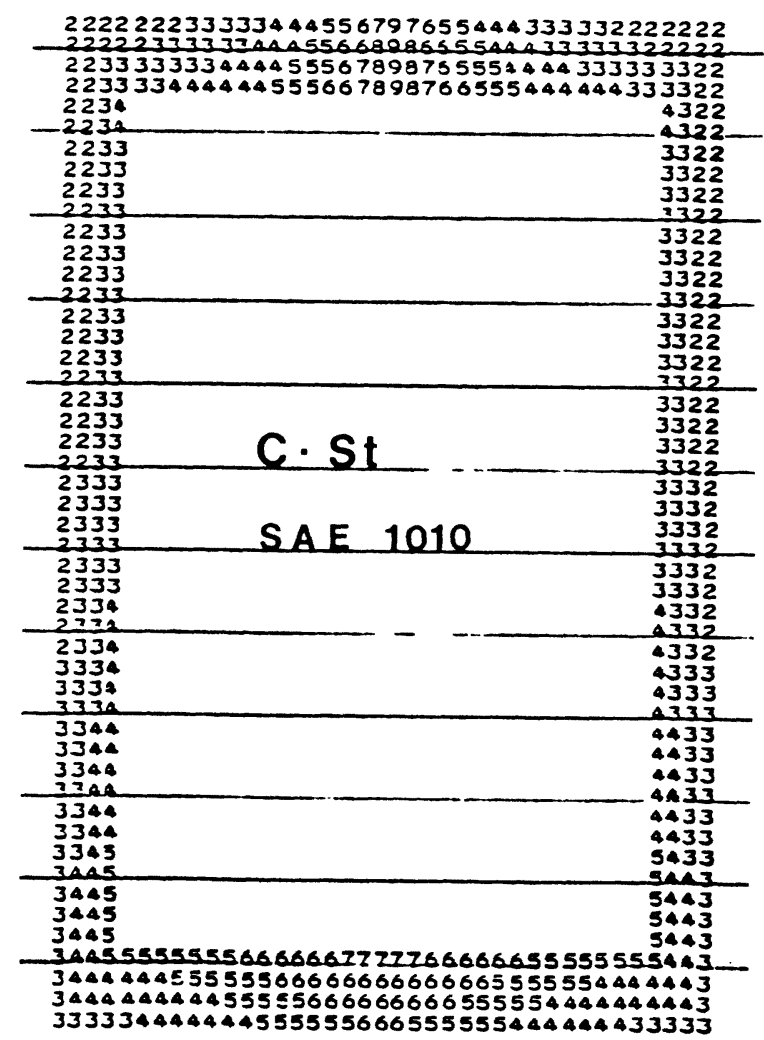

Fig. 11. Power loss density distribution in center arm elevated $0.58 \mathrm{~m}$.

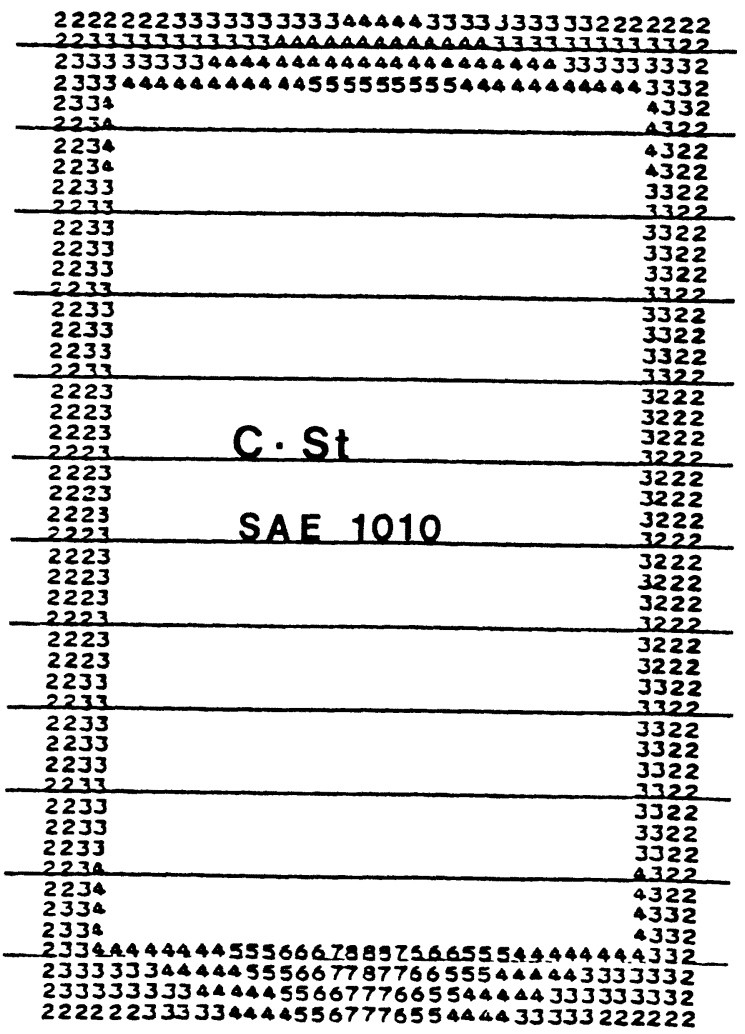

Fig. 12. Power loss density distribution in center arm elevated $0.97 \mathrm{~m}$.

each incremental elevation is also obtained. In addition, the computer program was extended to compute the temperatures as a function of plate material thickness and coolant flow. Fig. 15 shows the inside (coolant) and outside (air) 
TABLE I

NUMBERS ASSIGNED TO POWER LOSS DENSITY RANGES FOR COMPUTER PRINT OUT

\begin{tabular}{ccc}
\hline Number & $\begin{array}{c}\text { Upper } \\
\text { W/m }\end{array}$ & $\begin{array}{c}\text { Range } \\
\text { Lower } \\
\mathrm{W} / \mathrm{m}^{3}\end{array}$ \\
\hline 1 & $-{ }^{-} \times 10^{6}$ & $\leqslant 3.27 \times 10^{6}$ \\
2 & $1.20 \times 10^{6}$ & $0.442 \times 10^{6}$ \\
3 & $0.442 \times 10^{6}$ & $0.163 \times 10^{6}$ \\
4 & $0.163 \times 10^{6}$ & $0.060 \times 10^{6}$ \\
5 & $0.060 \times 10^{6}$ & $0.022 \times 10^{6}$ \\
6 & $0.022 \times 10^{6}$ & $0.0081 \times 10^{6}$ \\
7 & $0.0081 \times 10^{6}$ & $0.0030 \times 10^{6}$ \\
8 & $0.0030 \times 10^{6}$ & $0.0011 \times 10^{6}$ \\
9 & &
\end{tabular}

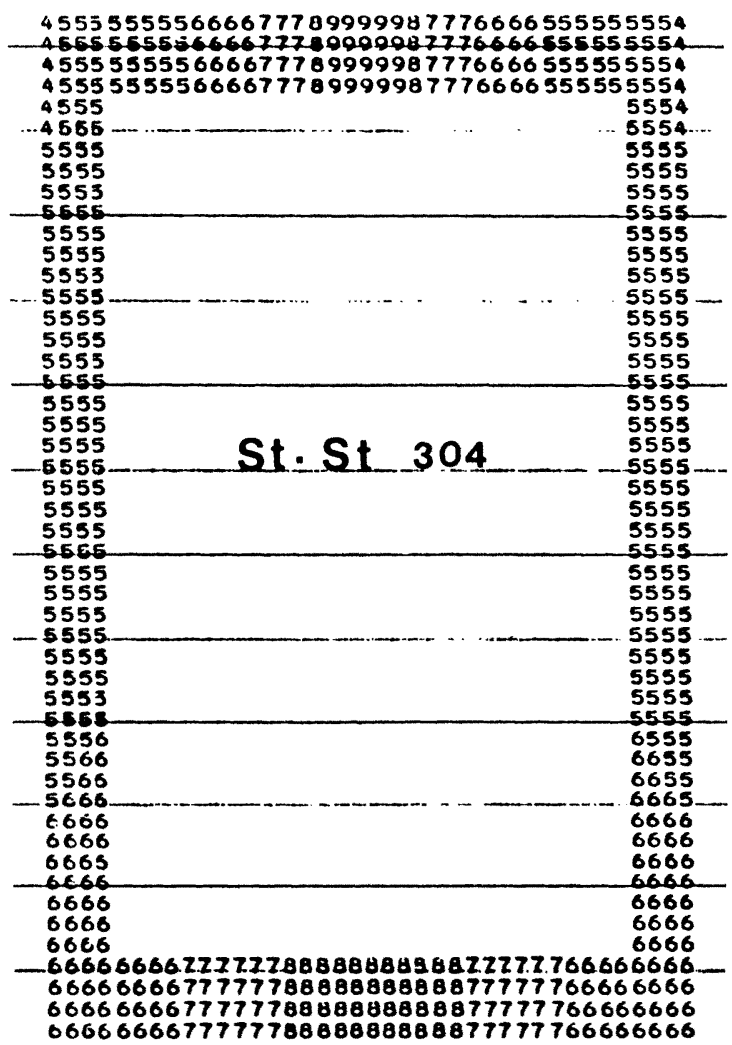

Fig. 13. Power loss density distribution in center arm elevated $0.58 \mathrm{~m}$.

surface temperatures as a function of the carbon steel material thickness for different coolant flows. The nominal flow of $0.6 \mathrm{ft} / \mathrm{s}$ corresponds to $40 \mathrm{gal} / \mathrm{min}$ for the coolant passage in question. Fig. 16 illustrates similar results except the material is stainless steel. For direct comparison between the two materials used for the specific material thickness in the design, Fig. 17 is included. It shows the outside surface temperature on the side of the center electrode arm at the elevation of $0.97 \mathrm{~m}$ as a function of the coolant flow rate. At smaller water flows, stainless steel attains a lower temperature due to much lower induced power losses. However, as can be seen with increasing flow rates the temperature difference becomes not only smaller but reverses, i.e., carbon steel has a lower surface operating temperature. This is mainly due to the better thermal conductivity of the carbon steel, showing

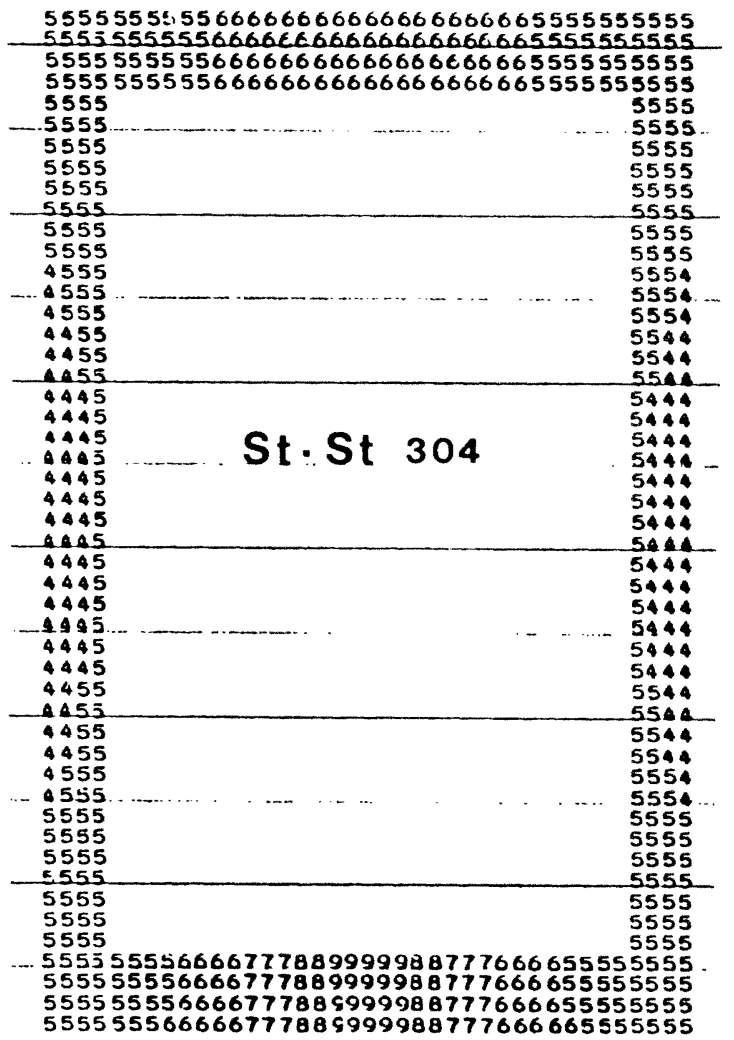

Fig. 14. Power loss density distribution in center arm elevated $0.97 \mathrm{~m}$.

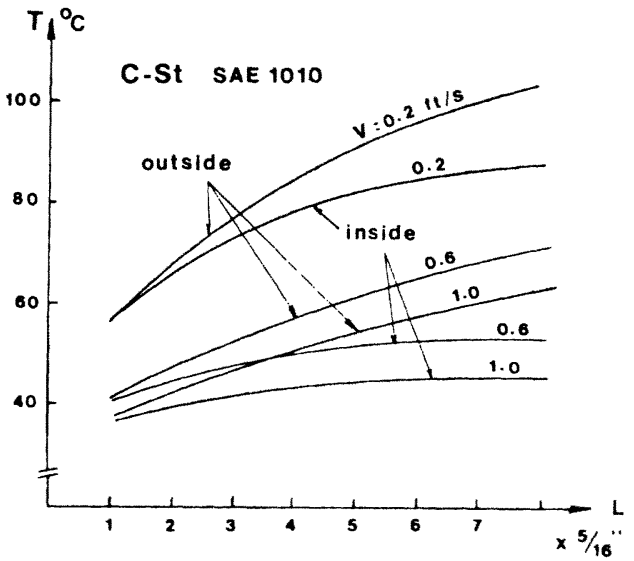

Fig. 15. Arm temperature versus plate material thickness for various flow rates.

that the induced heat is carried away more effectively by the coolant.

Some of the parameters used in the calculations for the induced losses and temperatures, either obtained from applicable data sheets, design specifications, calculations or emperically determined constants, are given in Table II.

\section{FIELD MEASUREMENTS}

Based on the results of this study, carbon steel was selected for construction of the electrode arms. It appeared that for the design criteria specified and expected operating conditions, the selection of stainless steel would only marginally lower operating temperatures. In view of the substantial 


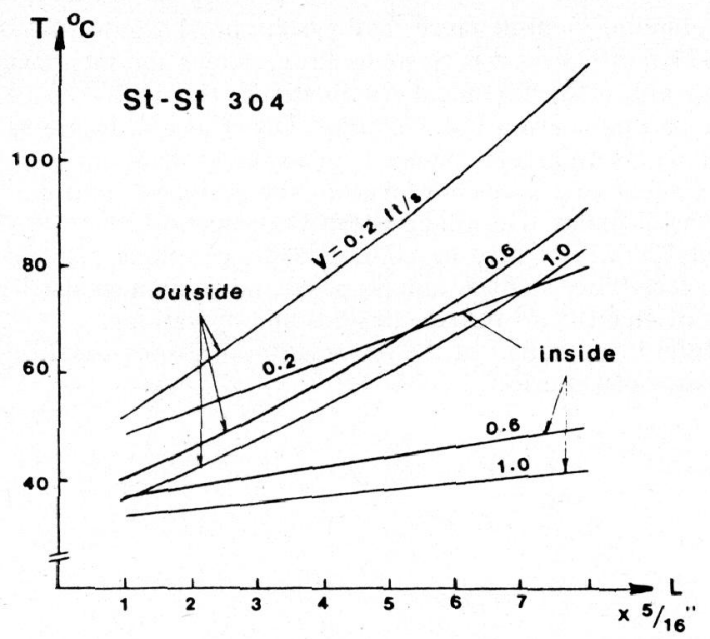

Fig. 16. Arm temperature versus plate material thickness for various flow rates.

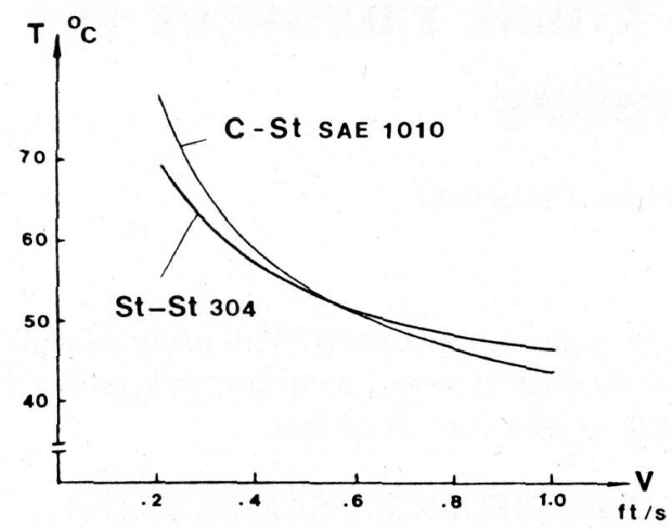

Fig. 17. Influence of material on electrode arm surface temperature.

savings in material costs, the use of stainless steel was therefore not warranted.

This conclusion proved correct and has been substantiated after five months of sucessful furnace operation. In addition, measurements on the center electrode arm discharge cooling-water system indicated water temperature rises not to exceed $38^{\circ} \mathrm{C}$ (compared to the intake) under the most severe furnace operating conditions. Furthermore, the use of stick-on temperature pads and an infrared temperature detector (Williamson 2200) indicated that surface temperatures resemble closely (in all circumstances within $11^{\circ} \mathrm{C}$ ) those predicted by the theory. This was true even for measurements taken at reduced flow rates when compared to corresponding predicted values.

\section{CONCLUSION}

The objective of the investigation to ensure, with the material selected and design criteria specified, that the center electrode arm could be operated in any relative position between the outside electrode arms (with the maximum phase current of $80 \mathrm{kA}$ ) without adverse effects from induced heating was accomplished.

\section{ACKNOWLEDGMENT}

The authors wish to thank E. R. Wunsche, President, EMPCO (Canada) Ltd. for his permission to publish the results.
TABLE II

PARAMETERS USED IN THE CALCULATIONS

\begin{tabular}{lcc}
\hline Symbol & C-St 1010 & St-St 304 \\
\hline$f(\mathrm{~Hz})$ & 60 & 60 \\
$\delta(\mathrm{cm})$ & 2.70 & 54.6 \\
$\alpha(\Omega-\mathrm{m})$ & $0.88 \times 10^{6}$ & $1.39 \times 10^{4}$ \\
$\mu_{r}$ & 100 & 1.02 \\
$h_{i}(\mathrm{~W} / \mathrm{mK})$ & $20-30$ & $20-30$ \\
$h_{o}(\mathrm{~W} / \mathrm{mK})$ & 686 & 686 \\
$l(\mathrm{~cm})$ & 1.59 & 1.59 \\
$k(\mathrm{~W} / \mathrm{mK})$ & 52 & 20 \\
$q_{0}^{\prime \prime \prime}\left(\mathrm{W} / \mathrm{m}^{3}\right)$ & $0.6 \times 10^{3}$ & $0.2 \times 10^{6}$ \\
$I(\mathrm{kA})$ & 80 & 80 \\
$T_{o}(\mathrm{~K})$ & 573 & 573 \\
$T_{i}(\mathrm{~K})$ & 300 & 300 \\
\hline
\end{tabular}

They are also grateful to W. Coleman, Chief Electrical Engineer, Chaparral Steel Co., Midlothian, TX for obtaining the experimental data on this furnace and for his cooperation throughout this investigation.

\section{REFERENCES}

[1] J. J. Trageser, "Induced heating in ultra high powered electric arc furnaces," in Proc. 1973 Electric Arc Furnace Conf., Houston, TX, p. 33.

[2] K. V. Namjoshi and P. P. Biringer, "Eddy current losses in magnetic beams close to a current carrying conductor," in Conf. Rec. 1978 Ind. Appl. Soc. Annu. Meeting.

[3] R. L. Stoll, Analysis of Eddy Currents. Oxford: Clarendon, 1974.

[4] M. Stafl, Electrodynamics of Electrical Machines. Czechoslovak Academy of Sciences. Iliffe. London, 1967.

[5] P. F. Ryff, "Eddy current losses in cylindrical arc furnace shells," paper to be published in IEEE Trans. Ind. Appl., May/June 1981.

[6] V. Arpaci, Conduction Heat Transfer. Reading, MA: Addison Wesley, 1966.

[7] F. Kreith, Principles of Heat Transfer. New York: Harper and Row, 1973.

[8] B. Gebhart, Heat Transfer. New York: McGraw-Hill, 1971.

[9] O. H. Mehus, "Induced losses and heating problems in high current arc furnace systems," Iron and Steel Engineer, May 1972.

[10] J. A. Ciotti, "A new era in melting," J. Metals, Apr. 1971.

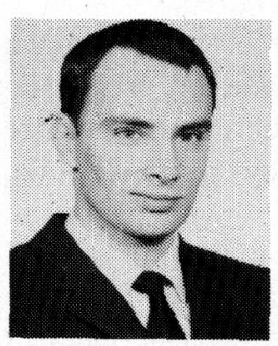

Peter F. Ryff (S'66-M'66-SM'78) was born in Amsterdam, The Netherlands. He received the Ingenieur degree in electrical engineering from the Engineering College in Amsterdam in 1958, and the B.A.Sc., M.A.Sc., and Ph.D. degrees in electrical engineering from the University of Toronto, Toronto, ON, Canada, in 1966, 1967 and 1969 , respectively.

From 1958 to 1964 he was employed by Honeywell, Inc., Toronto, and became their Engineering Field Representative. From 1969 to 1970 he was with General Time of Canada, Peterborough, ON, as a Research Engineer in the field of electromagnetic and solid-state timebase components. In 1970 he joined Ryerson Polytechnical Institute, Toronto, where he is Professor in the Electrical Technology Department. His research interests are in the fields of induction heating and arc melting furnaces.

Dr. Ryff is a member of the Association of Professional Engineers of the Province of Ontario and the American Society for Engineering Education. 


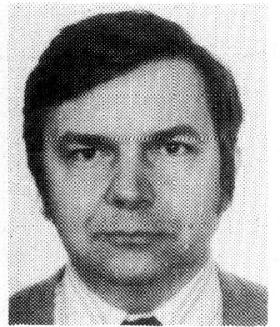

Vladimir Bulat received the B.S. degree and the M.S. degree in electrical engineering from the University of Zagreb, Yugoslavia, in 1960 and 1973 , respectively.

From 1960 to 1964 he was employed by a Yugoslavian company engaged in the design and installation of electric power distribution systems for industrial plants. From 1964 to 1974 he was with Zeljezara Sisak, Yugoslavia, where he held various engineering and management positions relating to electrical maintenance and electronic data processing. As the assistant to the superintendent of electrical maintenance for the entire plant, he was involved in all areas of electrical design, planning, maintenance, and system protection of the steel plant. From 1971 to 1974 he was Systems Programmer and then became Chief Systems Analyst for Technical Applications. From 1974 to 1977 he was with Ferrco Engineering Ltd., Whitby, ON, Canada, where he was their Power Systems Engineer engaged in power systems analysis and flow studies, protective device coordination, and design of distribution systems for mini-steel plants. He joined EMPCO (Canada) Ltd. in 1977 as Chief Electrical Engineer where he continued his activities in power systems analysis, feasibility studies, and proposal and equipment specifications in all areas of electric arc furnace designs and operations.

Mr. Bulat is a member of the Association of Professional Engineers of the Province of Ontario. 